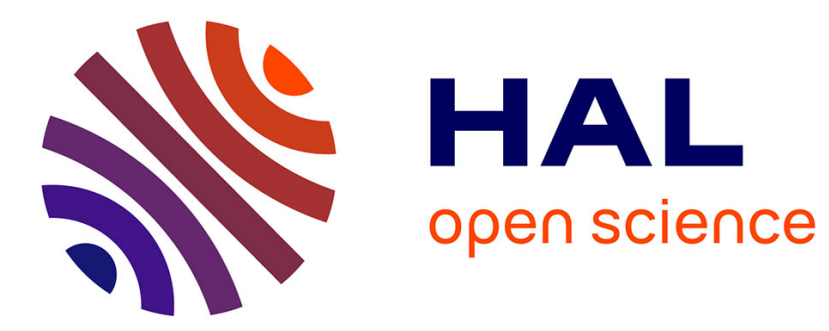

\title{
Wild-type and missense mutants of retinoschisin co-assemble resulting in either intra-cellular retention or incorrect assembly of the functionally active octomer
}

Lindsay J. Gleghorn, Dorothy Trump, Neil J. Bulleid

\section{- To cite this version:}

Lindsay J. Gleghorn, Dorothy Trump, Neil J. Bulleid. Wild-type and missense mutants of retinoschisin co-assemble resulting in either intra-cellular retention or incorrect assembly of the functionally active octomer. Biochemical Journal, 2009, 425 (1), pp.275-283. 10.1042/BJ20091179 . hal-00479241

\section{HAL Id: hal-00479241 \\ https://hal.science/hal-00479241}

Submitted on 30 Apr 2010

HAL is a multi-disciplinary open access archive for the deposit and dissemination of scientific research documents, whether they are published or not. The documents may come from teaching and research institutions in France or abroad, or from public or private research centers.
L'archive ouverte pluridisciplinaire HAL, est destinée au dépôt et à la diffusion de documents scientifiques de niveau recherche, publiés ou non, émanant des établissements d'enseignement et de recherche français ou étrangers, des laboratoires publics ou privés. 


\title{
Wild-Type and Missense Mutants of Retinoschisin Co-assemble Resulting in either Intra-cellular Retention or Incorrect Assembly of the Functionally Active Octomer
}

Lindsay J. Gleghorn ${ }^{1}$, Dorothy Trump ${ }^{2}$, Neil J Bulleid ${ }^{1,3 *}$

${ }^{1}$ Faculty of Life Sciences and ${ }^{2}$ Faculty of Medical and Human Sciences, University of Manchester, Manchester, U.K. ${ }^{3}$ Current address: Faculty of Biomedical and Life Sciences, University of Glasgow, Glasgow, UK

Address correspondence to: Neil J Bulleid, Faculty of Biomedical and Life Sciences, University of Glasgow, The Davidson Building, Glasgow, G12 8QQ. Tel +44 141 330 3027; fax +44 141330 5481; e-mail n.bulleid@bio.gla.ac.uk

\begin{abstract}
Abbreviations: The abbreviations used are: ER, endoplasmic reticulum; UPR, unfolded protein response; DTT, dithiothreitol; NEM, $N$-ethyl maleimide; BiP, immunoglobulin heavy chain binding protein; RS1, retinoschisin; DMEM, Dulbecco's modified Eagle's medium; PEI, polyethylimine; PMSF, phenylmethylsulphonyl fluoride; PSII, proteasome inhibitor II; endoH, endoglycosidase H; ECL, enhanced chemiluminescence; DAPI, 4',6-diamido-2phenylinimide; ERAD, ER-associated degradation.
\end{abstract}




\begin{abstract}
The X-linked disease retinoschisis is caused by mutations in the RS1 gene encoding retinoschisin, most commonly missense mutations leading to a lack of secretion of functional protein. One potential approach to treat this disease would be the introduction of the wild-type protein by gene therapy in affected individuals. Retinoschisin normally forms homooctamers so co-expression of the wild-type protein with the mutant could result in their co-assembly. Here we show that retinoschisin assembles into an octamer prior to transport from the endoplasmic reticulum and that co-assembly of wild-type and mutant protein can occur when they are co-expressed in the same cell. This co-assembly results in the retention of some but not all expressed wild-type retinoschisin. Moreover, when the wild-type protein is expressed with a missense mutant that is normally secreted co-assembly occurs resulting in the secretion of a heterogeneous mixture of oligomers. Missense mutations of retinoschisin which cause intracellular retention also lead to an unfolded protein response. However, this is not sufficient to decrease cell viability suggesting that the pathology of the disease is not likely to be linked to programmed cell death.
\end{abstract}

Keywords: retinoschisin, retinoschisis, intracellular retention, quality control, Xlinked disease, protein assembly.

\title{
Introduction
}

The endoplasmic reticulum (ER) of mammalian cells acts as a quality control checkpoint in the secretion of proteins, orchestrating correct folding and assembly and preventing the secretion of incorrectly folded or assembled molecules [1]. During the folding of proteins in normal healthy tissues this checkpoint is essential to both prevent the secretion of potentially toxic misfolded proteins and to conserve energy resources by degrading and recycling protein subunits of multi-subunit proteins that are made in excess. ER quality control also plays a crucial role in certain disease states (eg cystic fibrosis, emphysema, diabetes) where mutations in single proteins can lead to misfolding and intra-cellular retention thereby preventing secretion of the protein and hence cause loss of function [2]. Our understanding of the general mechanisms underlying the retention of misfolded proteins within the ER is limited to a well characterised system for the retention of glycoproteins by the ER lectins calnexin and calreticulin and a realisation that several ER resident proteins such as BiP can bind to exposed hydrophobic sequences in misfolded proteins and thereby retain these proteins within the ER [3]. Clearly if we are to develop rational therapies for such diseases our knowledge in this area needs to be strengthened, in particular when it comes to soluble non-glycoproteins linked to specific disease pathologies.

The X-linked disease retinoschisis is one such disease that results from inactivating mutations of the RS1 gene which encodes retinoschisin [4, 5]. Mutations are often missense leading to a lack of secretion of functional protein retinoschisin and resulting in splitting of the inner retinal layers, leading to visual deterioration in childhood [6]. Retinoschisin is a soluble non-glycosylated protein that is normally secreted from photoreceptor and bipolar cells of the retina [7]. Previously it was postulated to have a role in cell adhesion as it consists almost entirely of a single discoidin domain [8], however more recently it has been shown to associate with the alpha 1D subunit of the L-type voltage gated channels (L-VGCC $\alpha 1 \mathrm{D}$ ) regulating channel activity in a circadian manner [9] and the Na/K-ATPase/SARM1 complex 
[10]. Over 133 mutations in the gene coding for retinoschisin (RS1) are associated with X-linked retinoschisis, the majority being missense substitutions $[5,11]$. It is likely that these lead to loss of function as the disease is also caused by nonsense, deletions, insertions and splice-site mutations. Many of these mutations lead to intracellular retention of the mutated proteins presumably through aberrant folding or assembly [6, 8]. Retinoschisis is therefore an excellent example of a disease that is caused by the lack of secretion of a functional protein.

Current efforts to understand the molecular basis for retinoschisis have focused on an evaluation of the consequences of specific point mutations on disulphide bond formation and secretion of retinoschisin $[8,12]$. Retinoschisin is a $24 \mathrm{kDa}$ protein that contains 10 cysteine residues which form both intra- and inter-chain disulphide bonds and is normally secreted as a covalently linked homooctamer [12]. Formation of an octamer stabilised by inter-chain disulphide bonds is essential for function [12] and in this context it is interesting to note that $25 \%$ of the known missense mutations involve either a loss or gain of a cysteine residue. Studies to date have revealed that there are three major causes of loss of function of retinoschisin resulting from the missense mutations analysed; namely, mutations that prevent protein secretion, mutations that prevent oligomerisation but do not prevent secretion and mutations that do not prevent oligomerisation or secretion but presumably prevent interactions with partner proteins $[6,8]$.

In an effort to understand the disease pathology in more detail two knockout mouse models were created, both of which mimic the features of human X-linked retinoschisis $[13,14]$. When the RS1 gene was delivered to an adult knockout mouse the disease symptoms were reversed providing hope that delivery of wild-type RS1 to patients affected by retinoschisis may be an effective therapy [14, 15]. However, both disease models are not equivalent to the human patient as the mice were knockout models with no RS1 gene expression whereas affected individuals will be expressing mutant protein which may interfere with the ectopically expressed retinoschisin. A recent study has shown that wild-type protein can be secreted from cells when coexpressed with mutant proteins [16]. However, this study did not evaluate the ability of the mutant protein to co-assemble with wild-type within the cell. Here we address where oligomerisation of retinoschisin occurs, whether co-assembly of wild-type and mutant protein occurs within cells and whether the pathology of the disease can be explained by the retention of mutant protein causing an unfolded protein response (UPR) leading to cell death.

\section{Materials and Methods}

\section{Generation of epitope-tagged RS1 cDNA constructs}

PCR products containing the complete coding sequence for wild-type RS1 and the RS1 mutants C59S and C110Y were amplified from cDNA that had been generated previously [6]. To add an epitope tag, four forward primers were used in sequential rounds of amplification to add a V5 tag to the cDNAs following the N-terminal signal sequence after amino acid 23. Using the same method, a myc tag was added to the wild-type RS1 cDNA in the same position (primer sequences available on request). Once the full-length fragments had been generated, each was cloned using the pcDNA5/FRT/TO-TOPO vector by TA cloning (Invitrogen, Carlsbad, CA). The fulllength fragments were also digested from the pcDNA5 vector with PmeI and subcloned into pcDNA3.1 between the PmeI sites. 


\section{Generation of stable cell lines}

The T-REx ${ }^{\mathrm{TM}}$ system (Invitrogen, Carlsbad, CA) was used to create stable cell lines with tetracycline-regulated expression of retinoschisin. Plasmid DNA was transfected into HEK293 T-REx ${ }^{\mathrm{TM}}$ cells using Fugene6 (Roche, Indianapolis, IN, USA) according to the manufacturer's instructions. Stable cells were selected for 14 days with Hygromycin B (Invivogen, San Diego, CA) and Blasticidin (Invivogen, San Diego, CA). HEK293 T-REx ${ }^{\mathrm{TM}}$ cells expressing $\alpha 1$ antitrypsin were a kind gift from Lisa Swanton (University of Manchester, UK). All HEK293 T-REx ${ }^{\mathrm{TM}}$ cell lines were maintained in Dulbecco's modified Eagle's medium (DMEM) supplemented with $10 \%$ foetal calf serum and $2 \mathrm{mM} \mathrm{L}$-glutamine at $37^{\circ} \mathrm{C}$ with $5 \% \mathrm{CO}_{2}$.

\section{Cell culture and transfection}

COS-7 cells were maintained in DMEM supplemented with $10 \%$ foetal calf serum and $2 \mathrm{mM} \mathrm{L}$-glutamine at $37^{\circ} \mathrm{C}$ with $5 \% \mathrm{CO}_{2}$. For transfection, cells were seeded at about $80 \%$ confluence into $6 \mathrm{~cm}$ dishes. Transfections were performed using polyethylimine (PEI) (Polysciences, Eppelheim, Germany) at a ratio PEI:DNA of 2.5:1. Briefly, $4 \mu \mathrm{g}$ of plasmid DNA was mixed with $500 \mu 1$ DMEM (serum-free) and $10 \mu \mathrm{l}$ PEI $(1 \mu \mathrm{g} / \mu \mathrm{l})$. For co-transfections an equal amount of DNA was used for each construct. The transfection mixture was incubated at room temperature for $10 \mathrm{~min}$ before adding to the cells in $3.5 \mathrm{ml}$ DMEM plus $10 \%$ foetal calf serum.

\section{Pulse chase analysis}

Cells were grown to $80 \%$ confluence and gene expression was induced by incubating cells for $16 \mathrm{~h}$ with $10 \mu \mathrm{g} / \mathrm{ml}$ tetracycline. Cells were deprived of essential amino acids for $30 \mathrm{~min}$, incubated with radioactive methionine/cysteine protein labelling mix (50 $\mu \mathrm{Ci} / \mathrm{ml})$ (NEN, Boston, MA, U.S.A.) for a further $30 \mathrm{~min}$ and then the medium was replaced with DMEM with $10 \%$ foetal calf serum. Pulse chase analysis was carried out at $37^{\circ} \mathrm{C}$ unless otherwise indicated. When DTT was included in the pulse/chase, it was present at a final concentration of $5 \mathrm{mM}$. Cells were split into aliquots for required time points and at the required times cells and medium were separated and disulphide exchange was inhibited by the addition of $25 \mathrm{mM}$ NEM $(\mathrm{N}$ ethylmaleimide). Cells were lysed using lysis buffer $(50 \mathrm{mM}$ Tris/ $\mathrm{HCl}, 150 \mathrm{mM}$ $\mathrm{NaCl}, 2 \mathrm{mM}$ EDTA, $0.5 \mathrm{mM}$ phenylmethylsulphonyl fluoride (PMSF) and 1\% Triton $\mathrm{X}-100)$. Insoluble material was removed by centrifugation at $10000 \mathrm{xg}$ for $1 \mathrm{~min}$, and lysates were mixed with SDS to $1 \%(\mathrm{w} / \mathrm{v})$, boiled for $3 \mathrm{~min}$ and diluted 10-fold with lysis buffer. Clarified lysates were pre-cleared by incubation with Protein ASepharose for $30 \mathrm{~min}$. Immunoisolation was carried out by incubation with Protein A-Sepharose and anti-V5 antibody (Invitrogen, Carlsbad, CA) for $16 \mathrm{~h}$ at $4{ }^{\circ} \mathrm{C}$. Beads were washed three times with 100 vol RIPA buffer $(1 \%(\mathrm{w} / \mathrm{v})$ deoxycholic acid, $0.1 \%$ (w/v) SDS, $1 \%$ (v/v) Triton X-100, $50 \mathrm{mM}$ Tris- $\mathrm{HCl} \mathrm{pH} 8.0,500 \mathrm{mM} \mathrm{NaCl}$ and 0.5 $\mathrm{mM}$ PMSF) and resuspended in SDS-PAGE sample buffer (156.25 mM Tris-HCl, $\mathrm{pH}$ $6.8,1.5 \%$ SDS, $10 \%$ glycerol and bromophenol blue). Immunoisolation was repeated for whole media samples. For experiments where ER to Golgi transport was blocked, starvation and labelling were carried out at $37^{\circ} \mathrm{C}$ and the chase period was carried out at $15^{\circ} \mathrm{C}$. For experiments where the proteasome was blocked, cells were pre-incubated for 1 hour with $10 \mu \mathrm{M}$ Proteasome inhibitor II (PSII). PSII was added to cells throughout the starvation, labelling and chase period. For endoglycosidase $\mathrm{H}$ digestion immunoisolated material was treated with $500 \mathrm{U}$ of endo-H (New England Biolabs., Beverly, MA) at $37^{\circ} \mathrm{C}$ for 30 minutes, according to the manufacturer's instructions then analysed by SDS-PAGE. 


\section{Western blot analysis}

For western blot analysis, whole cell lysates were prepared by lysing transfected cells in 5x SDS loading buffer. For reducing SDS-PAGE 5x SDS sample buffer was supplemented with $50 \mathrm{mM}$ dithiothreitol (DTT). All samples were boiled for $5 \mathrm{~min}$ prior to loading on SDS-PAGE gels. Following electrophoresis, samples were transferred onto nitrocellulose membrane. The membrane was incubated with the primary antibody (1: 500 dilution for the RS1 [17], 1: 10000 for V5 and 1:3000 for the myc antibody [c-Myc A-14, Santa Cruz Biotechnology Inc]) for 1 hour and then the relevant secondary reagent (peroxidase-labelled goat anti-rabbit IgG antibody for the retinoschisin primary or peroxidase labelled protein A for V5 and myc antibodies) for 1 hour. The signal was visualised using enhanced chemiluminescence reagent (ECL, Pierce, Northumberland, UK), and exposed to X-ray film (FUJ, Dusseldorf, Germany).

\section{Sucrose gradient fractionation}

Approximately $10^{7}$ cells were lysed by vortex-mixing in sucrose gradient buffer (5 $\mathrm{mM}$ Tris/HCl, pH 7.5, $100 \mathrm{mM} \mathrm{NaCl}, 1 \mathrm{mM}$ PMSF and 0.1\% Nonidet P40) following treatment with $25 \mathrm{mM}$ NEM. Cleared lysates were applied to a continuous gradient of 9 parts of $5 \%(\mathrm{w} / \mathrm{v})$ sucrose into 9 parts of $25 \%(\mathrm{w} / \mathrm{v})$ sucrose, laid over 2 parts of $50 \%(\mathrm{w} / \mathrm{v})$ sucrose in sucrose gradient buffer. Gradients were centrifuged at 40000 $\mathrm{rpm}$ for $16 \mathrm{~h}$ at $4^{\circ} \mathrm{C}$ in a Beckman SW40Ti rotor and then separated into ten equalvolume fractions. Proteins were precipitated with $10 \%(\mathrm{v} / \mathrm{v})$ trichloroacetic acid and resuspended in SDS-PAGE sample buffer. For reducing, denaturing gradients, cleared lysates were supplemented with $1 \%(\mathrm{w} / \mathrm{v})$ SDS and $10 \mathrm{mM}$ DTT and boiled for $5 \mathrm{~min}$ prior to fractionation and gradients contained $0.1 \%$ SDS.

\section{Immunofluorescence}

COS-7 cells were grown on cover slips and transfected as described above. $48 \mathrm{~h}$ after transfection, cells were washed with phosphate buffered saline (PBS), fixed in ice cold methanol for $10 \mathrm{~min}$ at $-20^{\circ} \mathrm{C}$ and then washed again with PBS. Cells were then incubated at room temperature for $30 \mathrm{~min}$ with primary antibodies [1:300 dilution for V5 antibody and 1: 200 dilution for myc antibody]. After washing in PBS, cells were incubated at room temperature for $30 \mathrm{~min}$ with secondary antibodies [1:100 dilution for Alexa Fluor ${ }^{\circledR} 594$ anti-rabbit antibody (myc) and 1:100 dilution for Texas red anti-mouse antibody (V5)]. DNA was stained with 4',6-diamido-2-phenylinimide (DAPI).

\section{Co-immunoisolation}

Cells were lysed in lysis buffer (1\% (v/v) Triton X-100, $50 \mathrm{mM}$ Tris-HCl pH 7.4, 150 $\mathrm{mM} \mathrm{NaCl}, 2 \mathrm{mM}$ EDTA and $0.5 \mathrm{mM}$ PMSF). Clarified lysates were pre-incubated with protein-A Sepharose for $30 \mathrm{~min}$ to limit nonspecific binding, before incubation with anti-myc antibody conjugated to agarose beads (Upstate, Temecula, CA) overnight at $4{ }^{\circ} \mathrm{C}$. Beads were washed three times with lysis buffer before being resuspended and boiled for $2 \mathrm{~min}$ in SDS-PAGE sample buffer containing $50 \mathrm{mM}$ DTT. Immunoisolated material was separated by SDS-PAGE and analysed by western blot with the anti-V5 antibody.

\section{Luciferase reporter assay}

The px5ATF6-GL3 plasmid (UPR element luciferase reporter construct) was purchased from Addgene (Cambridge, MA). The pRL-TK plasmid (control renilla 
construct) was purchased from Promega, Southampton, UK. HEK293 T-REx ${ }^{\mathrm{TM}}$ cells were seeded into 6 well plates at $60 \%$ confluency and then co-transfected with $50 \mathrm{ng}$ pRL-TK and $400 \mathrm{ng}$ px5-ATF6-GL3 using LipfectAMINE 2000 (Invitrogen, Carlsbad, CA) according to the manufacturer's protocol. Expression of the gene of interest was induced by the addition of $10 \mu \mathrm{g} / \mathrm{ml}$ tetracycline for $16 \mathrm{~h}$. $24 \mathrm{~h}$ posttransfection cells were lysed and the luciferase activity was quantified using the DualLuciferase Assay system (Promega, Southampton, UK).

\section{Crystal Violet cell viability assay}

HEK293 T-REx ${ }^{\mathrm{TM}}$ cells were seeded into 6 well plates at $60 \%$ confluency. Expression of the gene of RS1 was induced by the addition of $10 \mu \mathrm{g} / \mathrm{ml}$ tetracycline. Detached cells were removed $48 \mathrm{~h}$ after the addition of tetracycline, by washing with PBS and the remaining adherent cells were fixed in methanol at $-20{ }^{\circ} \mathrm{C}$ for $5 \mathrm{~min}$. Cell nuclei were stained by the addition of $0.2 \%(\mathrm{w} / \mathrm{v})$ Crystal Violet and $2 \%(\mathrm{v} / \mathrm{v})$ ethanol for 10 minutes before washing 3 times with PBS. The stain was solubilised in $0.1 \%(\mathrm{w} / \mathrm{v})$ SDS, diluted 5-fold and quantified by measuring the absorbance at $540 \mathrm{~nm}$.

\section{Results}

\section{Assembly of retinoschisin into an octamer occurs in the ER}

It has been shown previously that retinoschisin exists as an octamer when isolated from cell culture medium or from tissues $[8,18]$. To investigate the potential coassembly of wild-type and mutant retinoschisin we first needed to determine exactly where in the cell this oligomerisation event occurred. To facilitate these studies we constructed stable cell-lines that over-express retinoschisin in a tetracycline inducible system. The cell lines constructed included wild-type and two disease causing mutants, C59S and C110Y. The C59S mutant does not assemble and is secreted as disulphide linked dimers whereas the $\mathrm{C} 110 \mathrm{Y}$ mutant is retained in the cell and does not assemble [8, 18] (see Fig. 1A for schematic of disulphide linkages). To aid detection of all constructs we included either a myc or V5 epitope tag after the signal sequence. The inclusion of an epitope tag at this position does not interfere with retinoschisin localisation, octamer formation or secretion [16].

We first carried out a pulse chase experiment to determine the kinetics of octamer formation. Cells expressing wild-type retinoschisin were induced with tetracycline and then pulse-labelled. Retinoschisin was isolated and separated under non-reducing conditions. No radiolabelled protein was seen in the absence of induction with tetracycline (Fig. 1B, lane 2). Several radiolabelled proteins were seen following induction. These include monomeric retinoschisin with the fastest mobility as well as slower migrating, higher order structures culminating with the slowest migrating band which corresponds to an octomer (Fig. 1B, lanes 3-8). The monomer migrated faster through the gel under non-reducing than reducing conditions (Fig. 1B, compare lanes land 3) indicating that intra-chain disulphides formed rapidly in newly synthesised retinoschisin. The presence of octamers immediately after the pulse (Fig. 1B, lane 3) suggests that assembly occurs rapidly and possibly in the ER. When the medium samples were analysed it was clear that only the octomer was secreted (Fig. 1B, lane 10). Hence the cell line is capable of both assembling octameric retinoschisin and contains an efficient quality control which prevents secretion of intermediates in the assembly pathway.

To pinpoint where in the cell assembly occurs we took advantage of the fact that ER to Golgi transport can be inhibited if the temperature of incubation is dropped to 
$15^{\circ} \mathrm{C}$. To verify that transport was prevented we analysed the maturation of endoglycosidase $\mathrm{H}$ resistant glycans in $\alpha 1$-antitrypsin, an event that occurs following modification of oligosaccharide side chains in the Golgi apparatus [19]. We showed that the $\alpha 1$-antitrypsin becomes resistant to endoglycosidase $\mathrm{H}$ digestion after 60 minutes of chase when the cells are incubated at $37^{\circ} \mathrm{C}$ but remains sensitive to digestion when incubated at $15^{\circ} \mathrm{C}$ (Fig. S1). When we carried out a similar experiment with wild type retinoschisin we saw assembly and secretion of the octomer into the medium when the cells were incubated at $37^{\circ} \mathrm{C}$ (Fig. 1C, lanes 1-5). When the cells were incubated at $15^{\circ} \mathrm{C}$, assembly into an octamer still occurred but this protein was not secreted (Fig. 1C, lanes 7-11). The fact that the octamer still forms demonstrates that assembly must occur in a pre-Golgi compartment which is presumably the ER.

\section{Some retinoschisin mutants can escape the ER quality control}

When we carried out a similar analysis to that for the wild type protein but with the C59S or C110Y mutant we obtained quite different results (Fig. 2). As expected, the C59S mutant failed to form octamers but was still secreted from cells predominantly as a dimer (Fig. 2A). The monomer formed intrachain disulphides as judged by its increased mobility compared to the fully reduced protein (Fig. 2A, compare lanes 1 and 4) indicating that the monomer at least had folded correctly. In contrast the C110Y mutant did not form oligomers and the monomer did not run as a single species, rather it formed a heterogonous mixture of redox forms that migrate between the fully reduced and fully disulphide bonded forms (Fig. 2B, compare lanes 1 and 3, marked with an asterisk). No retinoschisin was present in the medium indicating retention of this protein in the ER. The lack of correct folding of the monomer clearly precludes assembly. Moreover, the signal for the monomer disappeared over the time course which could indicate degradation of this protein or formation of high molecular weight aggregates that do not enter the gel (Fig. 2B top of resolving and stacking gel). To investigate whether the $\mathrm{C} 110 \mathrm{Y}$ mutant did form high molecular weight disulphide-linked complexes we also carried out the same pulse chase experiment but with DTT present in the pulse and chase to prevent disulphide formation (Fig. 2C). Reduced retinoschisin was present throughout the time course and was again absent from the medium. Some disappearance of retinoschisin was observed at later time points (Fig. 2C, lanes 7, 8) which indicates that some degradation occurred under these conditions.

The C59S mutant removes one of the cysteines involved in the formation of an interchain disulphide bond. Sucrose gradient fractionation was carried out in order to determine if non-covalent interactions were involved in the folding of retinoschisin (Fig. 3A). The octamer would be expected to fractionate at the highest sucrose concentration whereas the monomer would be expected to be at the lower sucrose concentrations. The fact that the protein is present throughout the gradient argues that interchain disulphides bonded intermediates in the assembly pathway do not form non-covalent interactions prior to the formation of the stabilising disulphide. In support of this conclusion, when the C59S mutant was fractionated under native conditions the protein was present at the lower sucrose concentrations (Fig. 3C) indicating that no octamer assembled. There was a slight shift in the fractionation profile to a higher sucrose concentration when compared to the wild-type or C59S samples that had been reduced and denatured prior to fractionation (Fig. 3B, D). This shift is likely due to the formation of disulphide linked dimers that can form between cysteine 40 in adjacent polypeptides (Fig. 1A) [8]. 


\section{Wild-type retinoschisin can co-assemble with mutant proteins}

Having established that assembly of the retinoschisin octamer occurs in the ER we then wanted to determine if wild-type and mutant protein could co-assemble when they were expressed together in the same cell. The approach we took was to cotransfect wild-type and mutant retinoschisin in COS-7 cells, a cell line that has been used previously to study retinoschisin co-assembly [16]. In these experiments the wild-type protein included either a myc or V5 epitope tag and the C59S or C110Y mutant contained a V5 epitope tag. To verify that the tags did not interfere with assembly and indeed that assembly and secretion of the octamer occurred in COS-7 cells we separated the media from transfected cells under non-reducing conditions and developed the resulting western blot with an antibody to retinoschisin (Fig. 4A). The wild-type V5 and wild-type myc formed octamers that were secreted from COS-7 cells. These octamers migrated slightly slower than the wild-type octamer presumably due to the presence of the epitope tag (Fig. 4A). We also established that we could cotransfect COS-7 cells with wild-type and mutant retinoschisin so that they were expressed in the same cell. When cells transfected with either wild-type V5 and wildtype myc or wild-type myc and V5-tagged mutants were analysed by immunofluorescence, signal for V5 and myc fluorescence was seen in the same cell (Fig. 4B).

To determine whether co-assembly occurred we co-transfected cells with wild-type myc and wild-type V5 or with wild-type myc and mutant V5. We included controls where cells were not transfected or were transfected with either wild-type myc or wild-type V5 on their own (Fig. 4E, F). We established that the transfections had been successful, by western blotting the cell lysates prior to immunoisolation (Fig. 4C, D). Following immunoisolation, no signal was seen in the cell lysate or medium from untransfected or singly transfected cells (Fig. 4E, F, lanes 1-3). A band corresponding to the size of retinoschisin was seen in the cell lysates for both the wild-type/wild-type and wild-type/mutant co-transfections (Fig. 4E, lanes 4-6) indicating co-assembly within the cell. However, only wild-type/wild-type and wild-type/C59S cotransfection resulted in the appearance of co-assembled retinoschisin in the cell medium (Fig. 4F, lanes 4 and 6). These results clearly demonstrate that the C110Y and the C59S mutant can co-assemble with the wild-type protein in the ER.

The co-assembled wild-type and C59S molecules that were secreted could potentially exist as dimers, octamers or intermediates between these two oligomers. When cells were co-transfected with wild-type myc and C59S V5 mutant retinoschisin and the medium samples immunoisolated with the myc antibody, several oligomeric intermediates were formed which cross-reacted with antibodies to the myc and V5epitope (Fig, 5A, B). The presence of V5 reactive oligomers and assembly intermediates shows that the C59S mutant co-assembles with the wild-type protein giving rise to the incorporation of the mutant protein into the oligomers. Moreover the oligomers formed between the C59S mutant and wild-type proteins were not subject to ER quality control and were secreted.

The co-assembly of wild-type and the C110Y mutant retinoschisin could lead to a block in secretion of wild-type protein. To determine if this was indeed the case we looked to see if wild-type protein was still secreted in these co-transfected cells. The cell lysates and medium samples from either singly transfected or co-transfected cells were analysed (Fig. 6). Wild-type protein was secreted from all cells that were transfected with wild-type protein even in the presence of the C110Y mutant (Fig. 6B, 
lanes 2, 4, 5). Hence, whilst some wild-type protein must be retained in the ER not all retinoschisin was prevented from being secreted when wild-type and the C110Y mutant were co-expressed.

\section{RS1 mutants and the UPR}

The time course experiments carried out with the C110Y mutant retinoschisin expressing cell line showed that the protein is retained in the ER and that there appears to be a disappearance of the protein over time. This could be due to the protein being degraded or forming larger aggregates that do not enter the SDS-PAGE gel. To determine if the protein is being degraded by ER associated degradation (ERAD) we carried out a pulse chase experiment but this time included the proteasomal inhibitor PSII to prevent ERAD (Fig. 6E). The samples were separated under reducing conditions. No disappearance of the radiolabel was seen either in the presence or absence of PSII. These results indicate that the C110Y mutant retinoschisin was not subjected to ERAD even though it is retained in the ER. This is in contrast to the situation when the $\mathrm{C} 110 \mathrm{Y}$ mutant is synthesised in the presence of DTT (see Fig 2C). Hence formation of a high molecular weight complex stabilised by disulphides prevents the protein being degraded by ERAD.

The retention of the $\mathrm{C} 110 \mathrm{Y}$ mutant and other mutant retinoschisin within the cell and the lack of degradation by ERAD is likely to induce a stress response that could lead to cell death. Such cell death could well contribute to the disease pathology alongside a lack of functional secreted protein. To assess whether the ER retention of retinoschisin caused an UPR we first constructed cell lines that expressed untagged wild-type and mutant retinoschisin in tetracycline-inducible HEK293 TREX cells. A reporter assay was used, which involved co-transfecting cells with a firefly luciferase gene under control of the ATF6 UPR element and a Renilla luciferase gene as a transfection efficiency control. The reporter assay system was validated by comparing UPR activity in untreated HEK293 TREX parent cells with that in tunicamycin treated HEK293 parent cells (Fig. 7A). Tunicamycin caused a 30-fold induction of UPR activity in tunicamycin treated cells compared to untreated cells.

The luciferase reporter assay was then used on cells expressing wild-type or mutant retinoschisin. There was a slight but significant UPR ( $\sim 2$ fold $)$ when the expression of wild-type or the C59S mutant retinoschisin was induced with tetracycline (Fig 7A) indicating that the overexpression of even correctly folded protein causes a stress response. However, a larger increase in UPR activity ( $\sim 8$ fold $)$ was measured following induction of expression of the $\mathrm{C} 110 \mathrm{Y}$ mutant retinoschisin (Fig. 7A). To determine whether the UPR induced by mutant $\mathrm{C} 110 \mathrm{Y}$ retinoschisin causes cell death, cell viability was investigated using a crystal violet assay (Fig. 7B). Only $10 \%$ of cells treated with tunicamycin were still viable after 48 hours, whereas cell lines that had been induced with tetracycline to express wild-type, C59S and C110Y retinoschisin showed no decrease in viability after 48 hours. These results demonstrate that while a strong UPR is elicited by expression of a mutant retinoschisin, this does not lead to cell death.

\section{Discussion}

It is now well established that the absence of functional, octameric retinoschisin causes the X-linked disease retinoschisis [20]. We show here that the assembly of this octameric protein occurs within the ER and that for the wild-type protein only the fully assembled molecule is secreted. Several intermediates in the assembly pathway 
are clearly present within cells yet none of these are secreted raising an interesting question as to how the ER quality control system retains these unassembled forms. Intriguingly the C59S mutant retinoschisin is secreted as a disulphide linked dimer demonstrating that the ER quality control system does not recognise and retain this particular dimeric species. Retinoschisin forms two interchain disulphides in the octamer, either between $\mathrm{C} 59$ and $\mathrm{C} 223$ or between two $\mathrm{C} 40$ residues on adjacent polypeptides (Fig. S1) [12]. It is possible that the presence of C59 causes retention of the wild-type protein until it is paired with C223. However, it has also been shown that a C223S mutant retinoschisin is also secreted from cells as a dimer [12] so it is not simply the presence of C59 that causes retention of the wild-type protein. If retention was via a thiol-mediated process, as has been shown previously for immunoglobulin $\mathrm{M}$ chain and adiponectin [21, 22], then the single cysteine mutants would be expected to be retained but they are not. Rather it seems that once the C59C223 disulphide is prevented from forming, then the cellular quality control does not retain the protein in the ER. It could be that the formation of the C59-C223 occurs very early in the maturation of retinoschisin and that once it is formed it is retained, possibly due to the exposure of a motif that is recognised by an ER resident protein. Secretion can only occur once the octamer is formed which would presumably mask the exposed motif. In the case of the mutants whereby the C59-C223 disulphide is prevented from forming the protein motif recognised by the ER quality control system is never exposed allowing secretion of a dimer stabilised by the C40-C40 disulphide.

Retinoschisis is an X-linked disease, so only one allele will ever be expressed in each cell as males are hemizygous and $\mathrm{X}$ inactivation means that females have only one $\mathrm{X}$ chromosome active in each cell. Hence there are no natural situations where mutant and wild-type protein would be expressed in the same cell. One of the potential mechanisms to treat the disease would be to introduce a wild-type gene into affected individuals by gene therapy. However, for this to be successful the wild-type protein would need to be secreted in a functional state when expressed in the presence of the mutant protein. Previously a report has been published which indicated that coexpression of wild-type and mutant retinoschisin did not prevent secretion of the wildtype protein [16]. This study did reveal that one particular retinoschisin mutant $(\mathrm{R} 141 \mathrm{H})$ did co-assemble with the wild-type protein and was secreted suggesting that individuals with this mutation are not likely to benefit from this therapeutic approach. Here we also show that the wild-type protein can be secreted when co-expressed with mutant retinoschisin. However, we also show that, in contrast to the previous report, the C59S mutant retinoschisin co-assembles with wild-type protein and this coassembled material is secreted. Remarkably it is not just dimers of mutant and wildtype protein that are secreted indicating that the mere presence of the C59S mutant subunit with multiple wild-type subunits is sufficient to subvert the ER quality control. It is highly unlikely that these partially assembled intermediates would be functional, so co-assembly may prevent the expression of functional retinoschisin. In addition the $\mathrm{C} 110 \mathrm{Y}$ mutant retinoschisin which is retained in the cell also coassembles with wild-type and retains some wild-type protein within the cell. It is likely that the level of expression of the wild-type protein relative to the mutant will determine how much protein is retained and consequently how much is secreted. It is difficult to predict the levels of expression of endogenous mutant protein in respect to ectopically expressed wild-type protein in a tissue, but our results should inform any potential gene therapy strategy. It could be that sufficient wild-type protein will be secreted in tissues even in the presence of mutant protein to alleviate the symptoms of 
the disease. An alternative strategy would be to target expression to cells which do not normally express retinoschisin such as Müller cells [23].

Finally, we demonstrate that the $\mathrm{C} 110 \mathrm{Y}$ mutant retinoschisin that is retained in the ER fails to form the correct intrachain disulphides yet is stable and forms high molecular mass aggregates that are stabilised by non-native interchain disulphides. The consequence of a lack of turnover of this mutant coupled to the fact that it is not folded correctly is that it induces the UPR; however, this does not lead to a decrease in cell viability. As retinoschisin is normally expressed in photoreceptor and bipolar cells of the retina any induction of programmed cell death would have drastic consequences for vision. Photoreceptor cell death does occur in retinoschisis [24] but this is thought to follow the formation of cysts and retinal splits $[14,15]$, which occur first, a phenotype that can be explained more realistically by a lack of cell adhesion. Hence our results support the concept that the disease pathology is caused simply by a lack of secretion of functionally active retinoschisin $[8,18]$.

\section{Acknowledgements}

We thank Lisa Swanton for the cell-line expressing $\alpha 1$-antitrypsin and Timothy Tavender for critical reading of the manuscript. The work was funded by a grant from the Medical Research Council reference G0501600.

\section{References}

1 Sitia, R. and Braakman, I. (2003) Quality control in the endoplasmic reticulum protein factory. Nature. 426, 891-894

2 Helenius, A. (2001) Quality control in the secretory assembly line. Philos. Trans. R. Soc. Lond. B Biol. Sci. 356, 147-150

3 Ellgaard, L., Molinari, M. and Helenius, A. (1999) Setting the standards: quality control in the secretory pathway. Science. 286, 1882-1888

4 Sauer, C. G., Gehrig, A., Warneke-Wittstock, R., Marquardt, A., Ewing, C. C., Gibson, A., Lorenz, B., Jurklies, B. and Weber, B. H. (1997) Positional cloning of the gene associated with X-linked juvenile retinoschisis. Nat. Genet. 17, 164-170

5 Consortium, T. R. (1998) Eunctional implications of the spectrum of mutations found in 234 cases with X-linked juvenile retinoschisis. The Retinoschisis Consortium. Hum. Mol. Genet. 7, 1185-1192

6 Wang, T., Waters, C. T., Rothman, A. M., Jakins, T. J., Romisch, K. and Trump, D. (2002) Intracellular retention of mutant retinoschisin is the pathological mechanism underlying X-linked retinoschisis. Hum. Mol. Genet. 11, 3097-3105

7 Molday, L. L., Hicks, D., Sauer, C. G., Weber, B. H. and Molday, R. S. (2001) Expression of X-linked retinoschisis protein RS1 in photoreceptor and bipolar cells. Invest Ophthalmol Vis. Sci. 42, 816-825

8 Wu, W. W. and Molday, R. S. (2003) Defective discoidin domain structure, subunit assembly, and endoplasmic reticulum processing of retinoschisin are primary mechanisms responsible for X-linked retinoschisis. J. Biol. Chem. 278, 28139-28146

9 Shi, L., Jian, K., Ko, M. L., Trump, D. and Ko, G. Y. (2009) Retinoschisin, a new binding partner for L-type voltage-gated calcium channels in the retina. J. Biol. Chem. 284, 3966-3975

10 Molday, L. L., Wu, W. W. and Molday, R. S. (2007) Retinoschisin (RS1), the protein encoded by the X-linked retinoschisis gene, is anchored to the surface of retinal photoreceptor and bipolar cells through its interactions with a Na/K ATPaseSARM1 complex. J. Biol. Chem. 282, 32792-32801 
11 Pimenides, D., George, N. D., Yates, J. R., Bradshaw, K., Roberts, S. A., Moore, A. T. and Trump, D. (2005) X-linked retinoschisis: clinical phenotype and RS1 genotype in 86 UK patients. J. Med. Genet. 42, e35

12 Wu, W. W., Wong, J. P., Kast, J. and Molday, R. S. (2005) RS1, a discoidin domain-containing retinal cell adhesion protein associated with X-linked retinoschisis, exists as a novel disulfide-linked octamer. J. Biol. Chem. 280, 1072110730

13 Weber, B. H., Schrewe, H., Molday, L. L., Gehrig, A., White, K. L., Seeliger, M. W., Jaissle, G. B., Friedburg, C., Tamm, E. and Molday, R. S. (2002) Inactivation of the murine X-linked juvenile retinoschisis gene, Rs1h, suggests a role of retinoschisin in retinal cell layer organization and synaptic structure. Proc. Natl. Acad. Sci. U. S. A. 99, 6222-6227

14 Zeng, Y., Takada, Y., Kjellstrom, S., Hiriyanna, K., Tanikawa, A., Wawrousek, E., Smaoui, N., Caruso, R., Bush, R. A. and Sieving, P. A. (2004) RS-1 Gene Delivery to an Adult Rs1h Knockout Mouse Model Restores ERG b-Wave with Reversal of the Electronegative Waveform of X-Linked Retinoschisis. Invest. Ophthalmol. Vis. Sci. 45, 3279-3285

15 Min, S. H., Molday, L. L., Seeliger, M. W., Dinculescu, A., Timmers, A. M., Janssen, A., Tonagel, F., Tanimoto, N., Weber, B. H., Molday, R. S. and Hauswirth, W. W. (2005) Prolonged recovery of retinal structure/function after gene therapy in an Rs1h-deficient mouse model of x-linked juvenile retinoschisis. Mol. Ther. 12, 644651

16 Dyka, F. M. and Molday, R. S. (2007) Coexpression and interaction of wildtype and missense RS1 mutants associated with X-linked retinoschisis: its relevance to gene therapy. Invest Ophthalmol Vis. Sci. 48, 2491-2497

17 Grayson, C., Reid, S. N., Ellis, J. A., Rutherford, A., Sowden, J. C., Yates, J. R., Farber, D. B. and Trump, D. (2000) Retinoschisin, the X-linked retinoschisis protein, is a secreted photoreceptor protein, and is expressed and released by WeriRb1 cells. Hum. Mol. Genet. 9, 1873-1879

18 Wang, T., Zhou, A., Waters, C. T., O'Connor, E., Read, R. J. and Trump, D. (2006) Molecular pathology of $X$ linked retinoschisis: mutations interfere with retinoschisin secretion and oligomerisation. Br. J. Ophthalmol. 90, 81-86

19 Lodish, H. F., Kong, N., Snider, M. and Strous, G. J. (1983) Hepatoma secretory proteins migrate from rough endoplasmic reticulum to Golgi at characteristic rates. Nature. 304, 80-83

20 Molday, R. S. (2007) Focus on molecules: retinoschisin (RS1). Exp. Eye. Res. 84, 227-228

21 Wang, Z. V., Schraw, T. D., Kim, J. Y., Khan, T., Rajala, M. W., Follenzi, A. and Scherer, P. E. (2007) Secretion of the adipocyte-specific secretory protein adiponectin critically depends on thiol-mediated protein retention. Mol. Cell Biol. 27, 3716-3731

22 Alberini, C. M., Bet, P., Milstein, C. and Sitia, R. (1990) Secretion of immunoglobulin $\mathrm{M}$ assembly intermediates in the presence of reducing agents. Nature. $347,485-487$

23 Takada, Y., Fariss, R. N., Tanikawa, A., Zeng, Y., Carper, D., Bush, R. and Sieving, P. A. (2004) A retinal neuronal developmental wave of retinoschisin expression begins in ganglion cells during layer formation. Invest. Ophthalmol. Vis. Sci. 45, 3302-3312 
24 Kirsch, L. S., Brownstein, S. and de Wolff-Rouendaal, D. (1996) A histopathological, ultrastructural and immunohistochemical study of congenital hereditary retinoschisis. Can. J. Ophthalmol. 31, 301-310

\section{Figure legends}

Figure 1: Folding and assembly of wild-type octameric retinoschisin occurs in the ER

(A) Schematic adapted from [12] depicting the arrangement of the known disulphides in retinoschisin. Only two subunits from the eight are shown, linked by interchain disulphides between two cys 40 residues and residues cys59 and cys 223 in adjacent subunits. Cys 83 is unpaired. It is not known whether the two remaining cysteines (cys38 and cys42) form a disulphide so are not included. (B) tetracycline-inducible HEK293 TREX stable cell line expressing V5-tagged wild-type retinoschisin was induced with $10 \mu \mathrm{g} / \mathrm{ml}$ tetracycline for 16 hours. After a 30 minute pulse, cells were chased for the indicated times. Non-induced cells were pulsed and chased for 160 mins (NI, lane 2). Aliquots of either cell lysates (lanes 1-8) or cell medium (lanes 9, 10) were subjected to immunoisolation with anti-V5 antibody and purified proteins analysed by $6-20 \%$ gradient SDS-PAGE either with (lanes 1,9 ) or without (lanes 2-8, 10) prior reduction. The position of the monomer $(\mathrm{m})$ and octamer $(\mathrm{o})$ is indicated. (C) A tetracycline-inducible HEK293 TREX stable cell line expressing myc-tagged wild-type retinoschisin was induced with $10 \mu \mathrm{g} / \mathrm{ml}$ tetracycline for 16 hours. After a 30 minute pulse, cells were chased for the indicated times. Chase was carried out at $37^{\circ} \mathrm{C}$ or $15^{\circ} \mathrm{C}$ as indicated. Aliquots of cell lysates and a sample of the cell medium at 120 minutes $(120 \mathrm{M})$ were subjected to immunoisolation with anti-myc antibody and isolated proteins analysed by $6-20 \%$ gradient SDS-PAGE under non-reducing conditions.

Figure 2: The C59S mutant is secreted whereas the C110Y mutant retinoschisin is retained in the cell.

A tetracycline-inducible HEK293 TREX stable cell line expressing V5-tagged C59S mutant retinoschisin (A) or C110Y mutant retinoschisin $(\mathbf{B}, \mathbf{C})$ was induced with 10 $\mu \mathrm{g} / \mathrm{ml}$ tetracycline for 16 hours. After a 30 minute pulse, cells were chased for the indicated times. $\mathrm{C} 110 \mathrm{Y}$ mutant was also pulse/chased in the presence of $5 \mathrm{mM}$ DTT (C). Non-induced C59S V5 HEK293 TREX stable cells were pulsed and chased for $160 \mathrm{~min}(\mathrm{NI})$. Aliquots of either cell lysates (lanes 1-8) or cell medium (lanes 9, 10) were subjected to immunoisolation with anti-V5 antibody and purified proteins analysed by $6-20 \%$ gradient SDS-PAGE either with (lanes 1,9 ) or without (lanes 2-8, 10) prior reduction. The position of the monomer (m) and dimer (d) is indicated. The mobility of $\mathrm{C} 110 \mathrm{Y}$ retinoschisin monomer is indicated with an asterisk.

Figure 3: Retinoschisin octomers are not stabilised by non-covalent interactions

Cell lysates from tetracycline-inducible HEK293 TREX stable cell lines expressing V5-tagged wild-type or C59S mutant retinoschisin that had been induced with 10 $\mu \mathrm{g} / \mathrm{ml}$ tetracycline for $16 \mathrm{~h}$ were subjected to sucrose gradient centrifugation under native or denaturing conditions. Following fractionation of the gradients, samples were precipitated with trichloroacetic acid and then separated by reducing SDS-PAGE before western blotting with the V5 antibody.

Figure 4: Co-assembly occurs between co-transfected wild-type and mutant retinoschisin 
(A) COS-7 cells were transfected with either untagged wild-type, V5- or Myc-tagged wild-type retinoschisin. 48 hours post-transfection, media was separated by $4-20 \%$ gradient SDS-PAGE under non-reducing (upper panel) or reducing (lower panel) conditions. Gels were analysed by Western blot using an anti-retinoschisin antibody. (B) COS-7 cells were transfected with the indicated constructs and $48 \mathrm{~h}$ later were fixed and stained with antibodies against the V5 or Myc epitope tags as indicated. (CF) COS-7 cells were untreated (lane 1) or transfected with either wild-type tagged with a myc or V5 epitope (WT myc; WT V5) alone (lanes 2, 3), together (lane 4) or wild-type tagged with the myc epitope was transfected with V5-tagged C59S (C59S V5) or $\mathrm{C} 110 \mathrm{Y}$ (C110Y V5) mutant retinoschisin (lanes 5 and 6). To check for expression whole cell lysates were separated by 10\% SDS-PAGE under reducing conditions and western blotted with antibodies against either the myc epitope tag (C) or the V5 epitope tag (D). Cell lysates (E) and cell media (F) was subjected to immunoisolation with an agarose-conjugated antibody to the myc epitope. The resulting immunoisolated material was separated by SDS-PAGE followed by western blotting with the anti-V5 antibody.

Figure 5: Wild-type and C59S mutant retinoschisin co-assemble to form oligomers that are secreted

COS-7 cells were untreated (lane 1 and 5) or transfected with either wild-type tagged with the myc epitope (WT myc) or C59S mutant retinoschisin (C59S V5) alone (lanes $2,3,6,7$ ) or together (lane 4 and 8 ). The cell media was subjected to immunoisolation with an agarose-conjugated antibody to the myc epitope. The resulting immunoisolated material was separated by SDS-PAGE through a 6-20\% gradient gel followed by western blotting initially with the myc antibody (A). The blot was stripped by brief treatment with $0.2 \mathrm{M} \mathrm{NaOH}$ before being reprobed with the V5 antibody (B).

Figure 6: Wild-type retinoschisin is still secreted when co-expressed with the C110Y mutant

COS-7 cells were untreated (lane 1) or transfected with either wild-type tagged with a myc or V5 epitope (WT myc; WT V5) alone (lanes 2, 3), together (lane 4) or wildtype tagged with the myc epitope was transfected with V5-tagged C110Y (C110Y V5) mutant retinoschisin (lane 6). Cell lysates $(\mathbf{A}, \mathbf{C})$ or media $(\mathbf{B}, \mathbf{D})$ were separated by $10 \%$ SDS-PAGE under reducing conditions and then western blotted with antibodies against either the myc epitope tag $(\mathbf{A}, \mathbf{B})$ to confirm WT RS1 expression in the cell lysate (A) and wild-type retinoschisin secretion into the media (B) or the V5 epitope tag to confirm mutant retinoschisin expression in the cell lysate (C) and mutant retinoschisin secretion into the media (D). (E) Tetracycline-inducible HEK293 TREX stable cell lines expressing V5-tagged C110Y mutant retinoschisin were induced with $10 \mu \mathrm{g} / \mathrm{ml}$ tetracycline for 16 hours. Cells were pre-incubated for 1 hour in complete media in the absence or presence of $10 \mu \mathrm{M}$ proteasome inhibitor II (PSII). Pulse chase was then carried out in the presence or absence of $10 \mu \mathrm{M}$ PSII. After a 30 minute pulse, cells were chased for the indicated times. Aliquots of cell lysates were immunoisolated with anti-V5 antibody and analysed by $12.5 \%$ reducing SDS-PAGE. Radiolabelled proteins were visualised by autoradiography.

Figure 7: Mutant retinoschisin induces the unfolded protein response but not cell death 
(A) Tetracycline-inducible HEK 293 TREX stable cell lines expressing wild-type retinoschisin (WT RS1) or the C59S or C110Y mutant retinoschisin (C59S RS1; C110Y RS1) or the parental cell line (HEK293 TREX) were transiently transfected with a reporter construct containing five UPR elements driving firefly luciferase (p5xATF6) and an internal control construct driving renilla luciferase (pRL-TK). $4 \mathrm{~h}$ after transfection, media was changed to either complete media or complete media containing $2 \mu \mathrm{g} / \mathrm{ml}$ tunicamycin (for the parental cell line) or tetracycline $10 \mu \mathrm{g} / \mathrm{ml}$ (for the cell lines expressing retinoschisin) for 16 hours. Luciferase activity was assessed 24 hours post-transfection. Results are represented as means $+\mathrm{SD}$ (error bars) of the firefly/renilla ratio expressed as relative luciferase units. Each assay was performed in triplicate and each experiment was repeated four times. $* * *=P<0.001$.

(B) HEK293 TREX parent cell lines were either treated with $2 \mu \mathrm{g} / \mathrm{ml}$ tunicamycin for 16 hours or left untreated. Tetracycline-inducible HEK 293 TREX stable cell lines expressing wild-type, C110Y or C59S mutant retinoschisin were induced for 16 hours with $10 \mu \mathrm{g} / \mathrm{ml}$ tetracycline or were untreated. $48 \mathrm{~h}$ after treatments, a crystal violet cell viability assay was performed. Results are represented as $\%$ cell viability \pm SD (error bars) of each treated cell line compared to untreated. Each assay was performed in triplicate. 


\section{Figure 1}

A

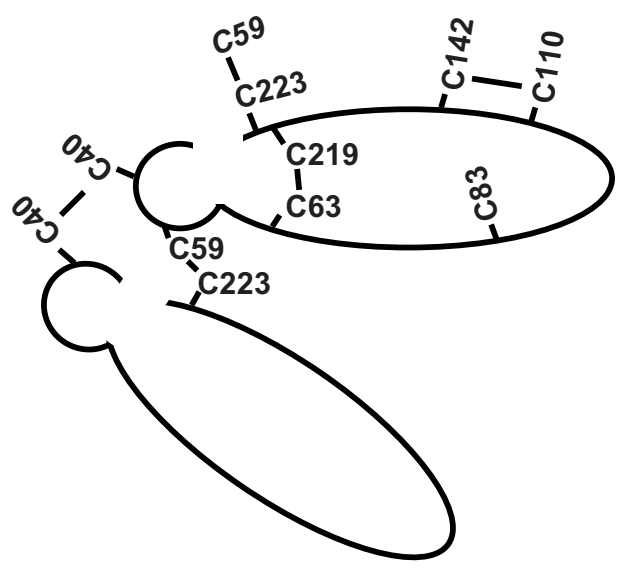

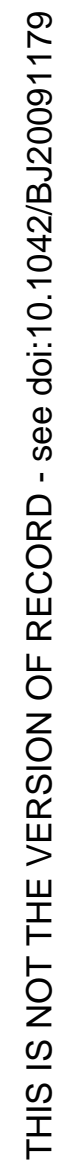

B

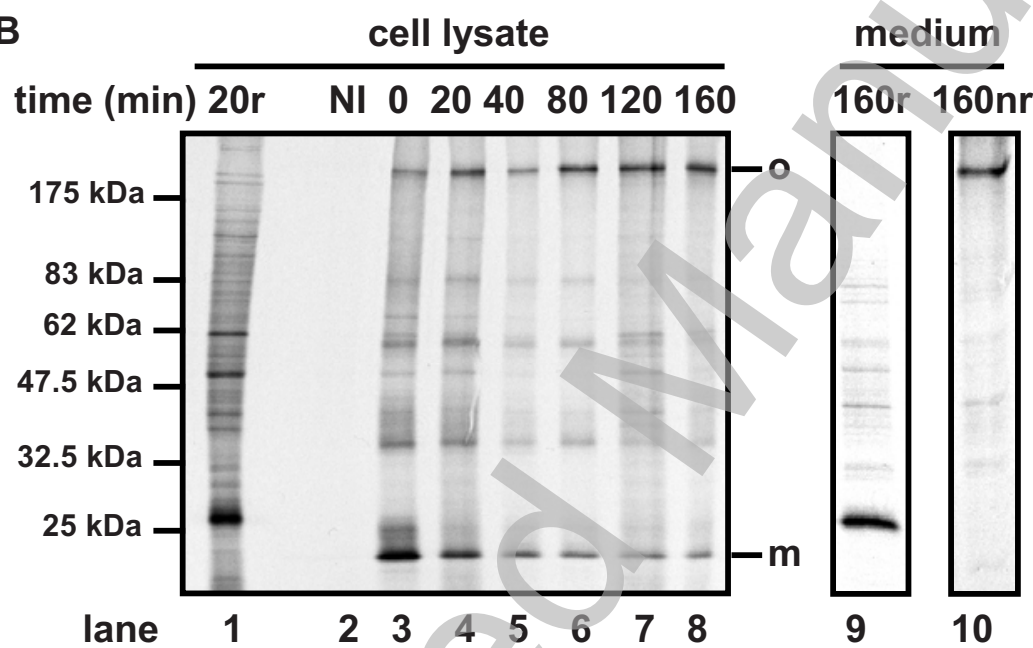

C

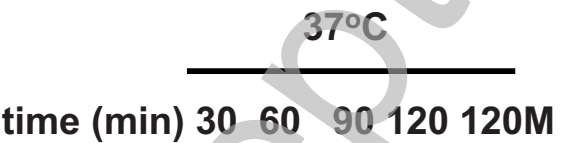

$15^{\circ} \mathrm{C}$

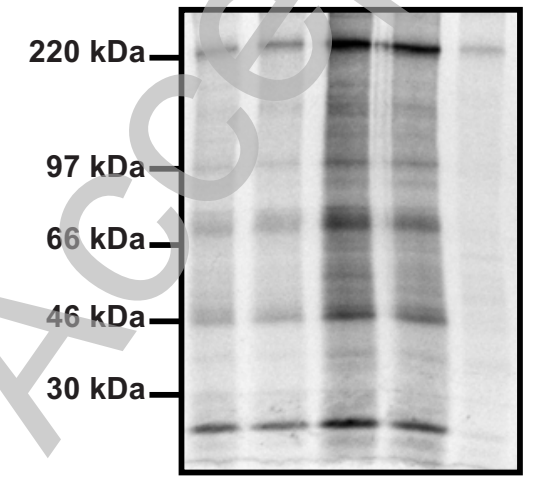

lane $\quad \begin{array}{lllll}1 & 2 & 3 & 4 & 5\end{array}$

$306090120120 \mathrm{M}$

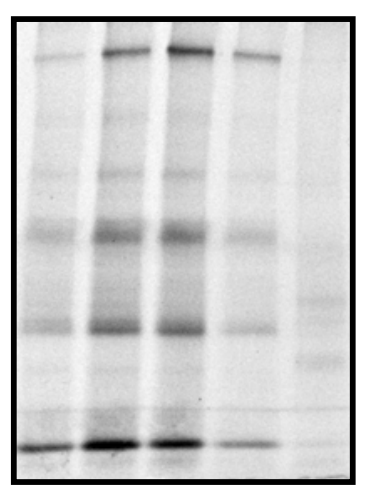

$\begin{array}{lllll}7 & 8 & 9 & 10 & 11\end{array}$ 
Figure 2
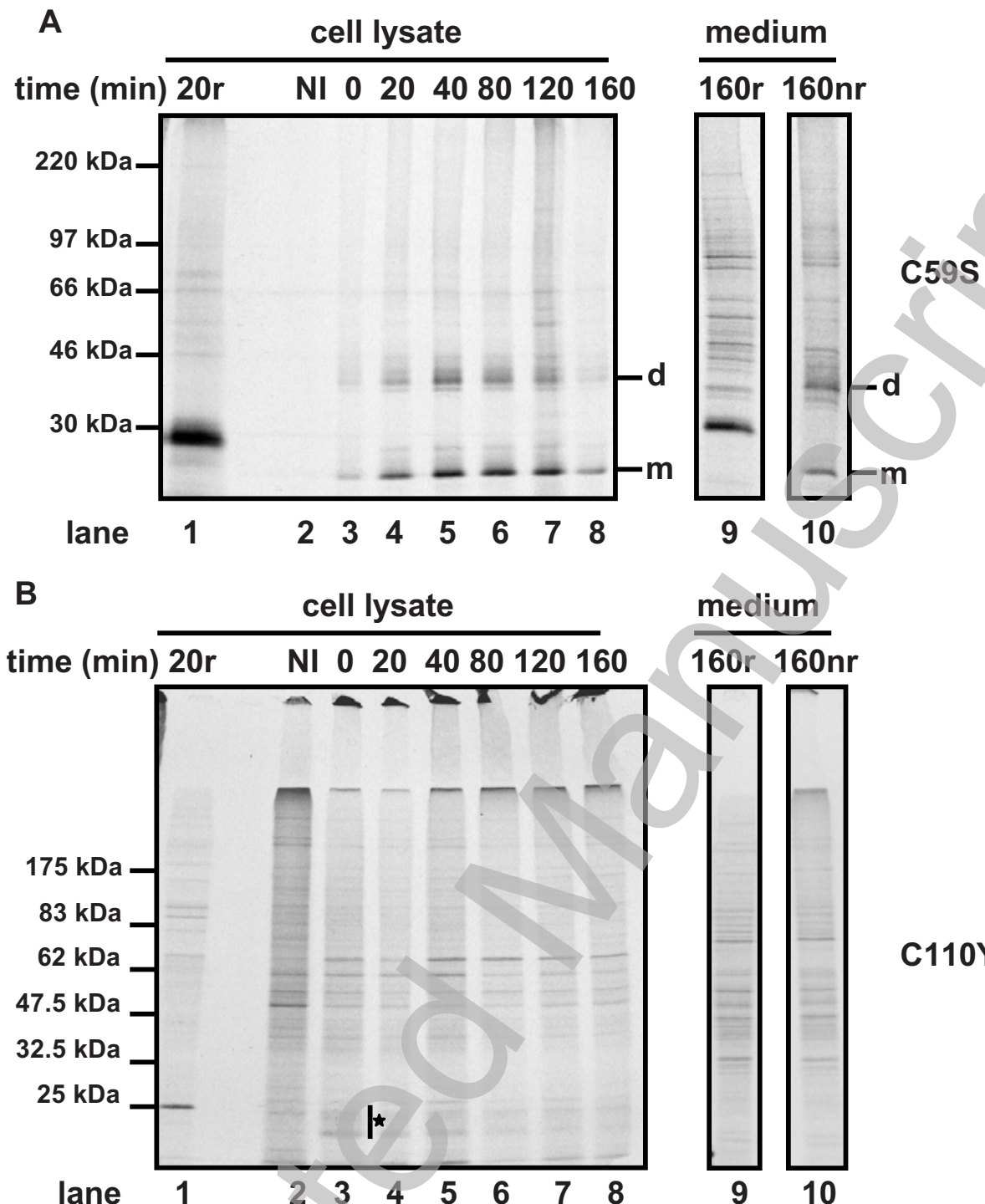

C110Y
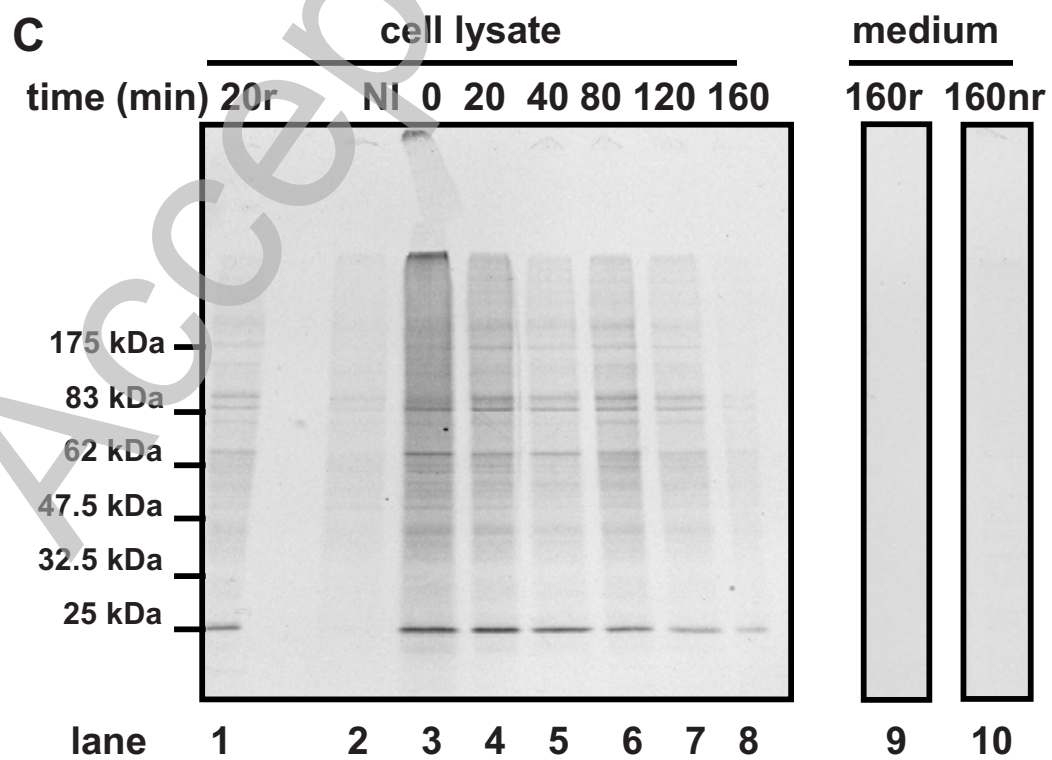

C110Y plus DTT in pulse/chase 
Figure 3

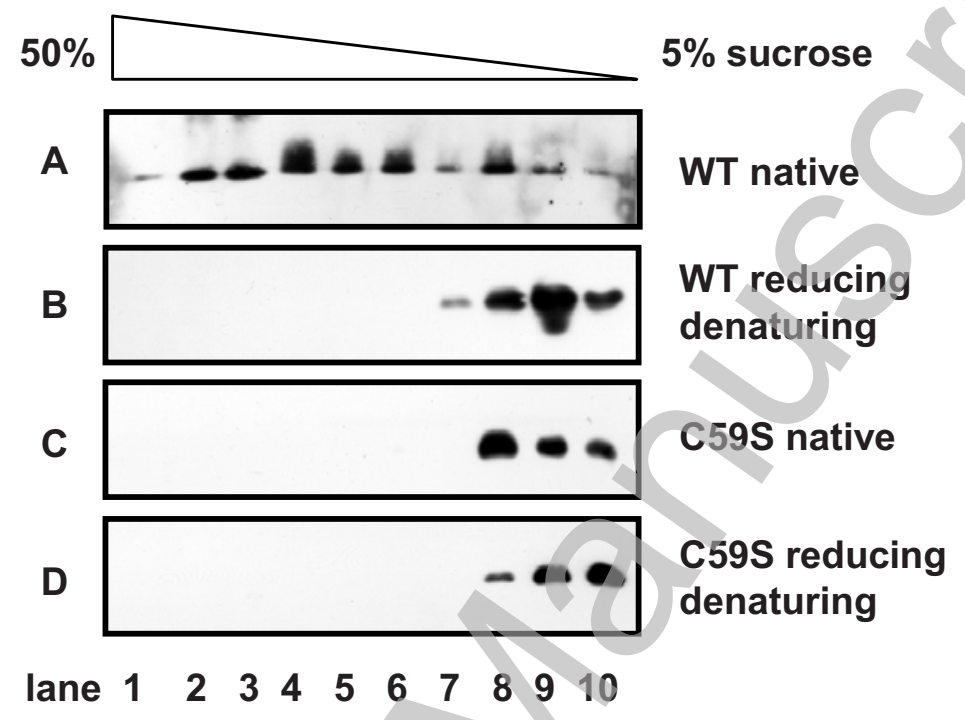


Biochemical Journal Immediate Publication. Published on 22 Oct 2009 as manuscript BJ20091179

Figure 4

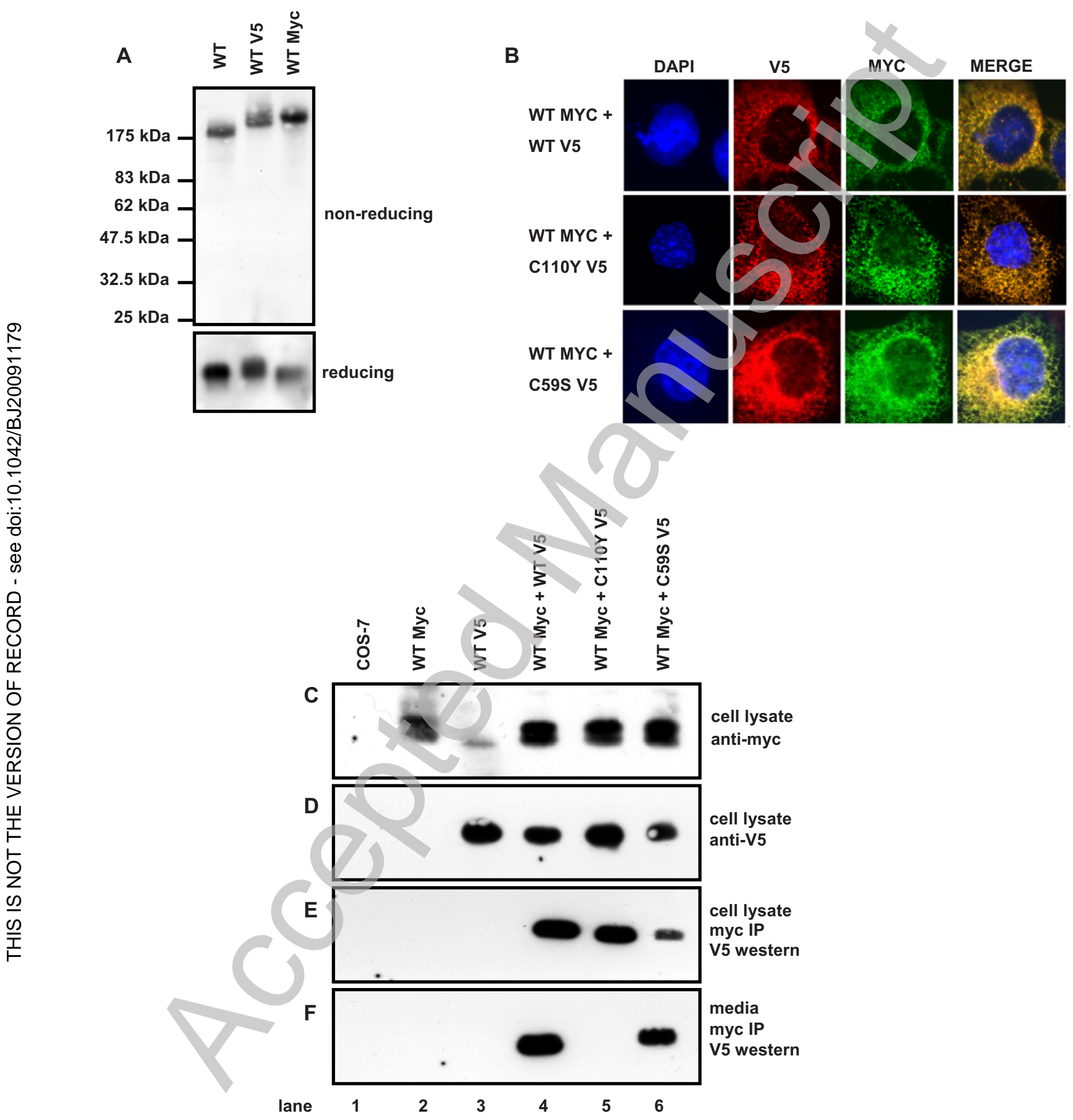

B

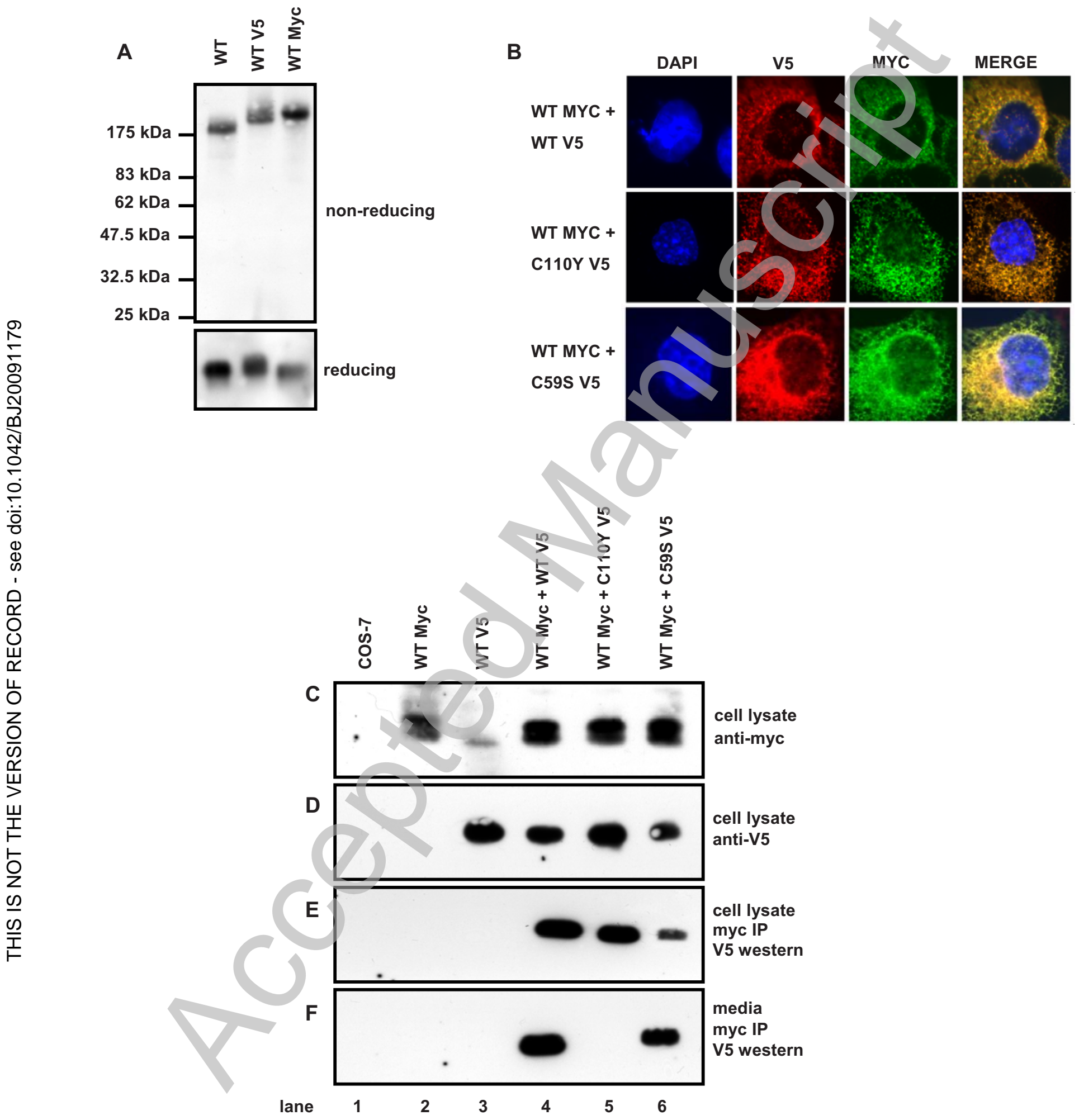

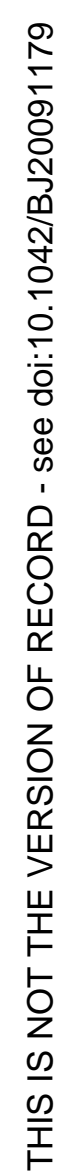

Licenced copy. Copying is not permitted, except with prior permission and as allowed by law. (C) 2009 The Authors Journal compilation (c) 2009 Portland Press Limited 


\section{Figure 5}

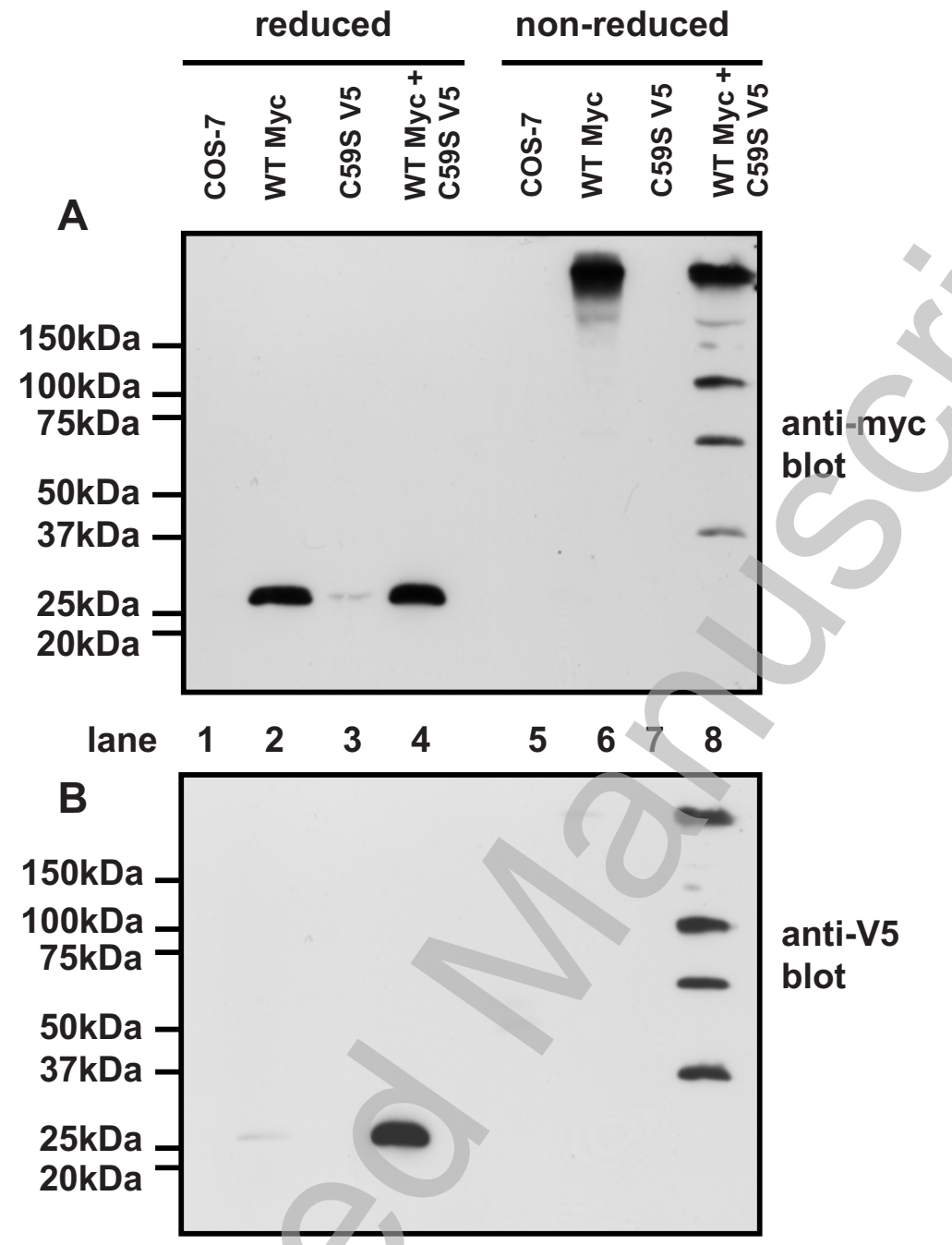




\section{Figure 6}

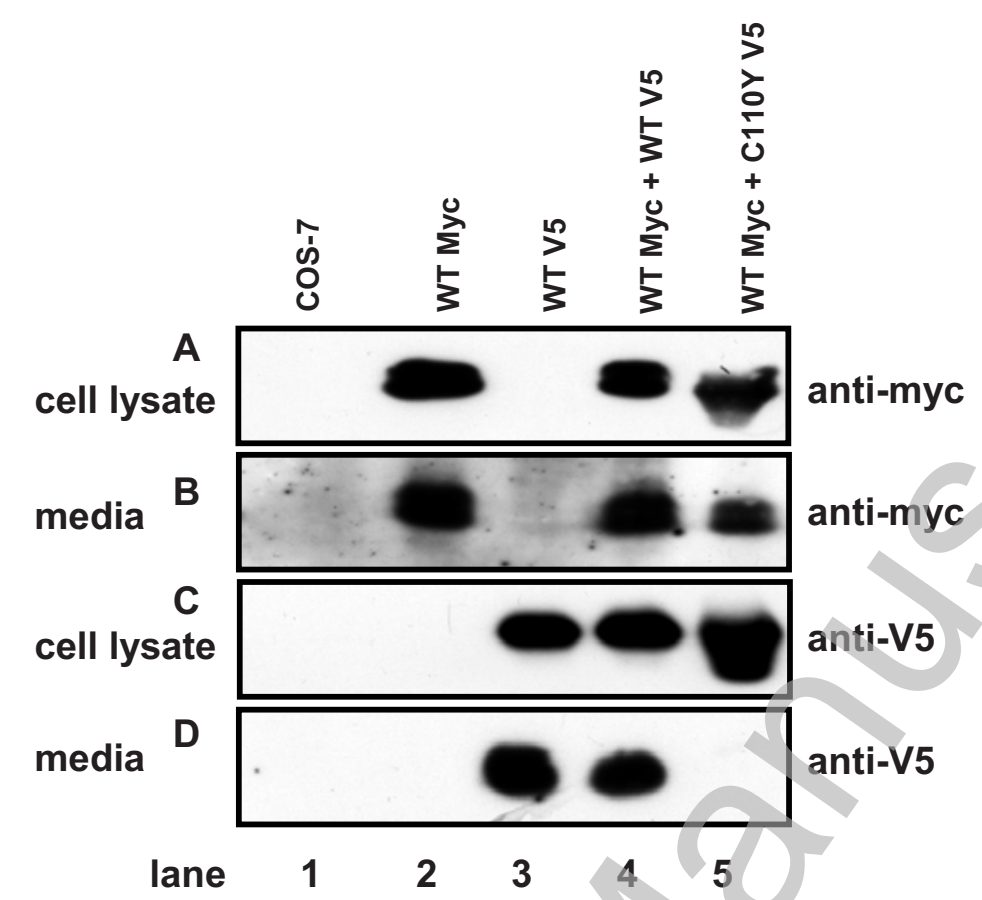

E
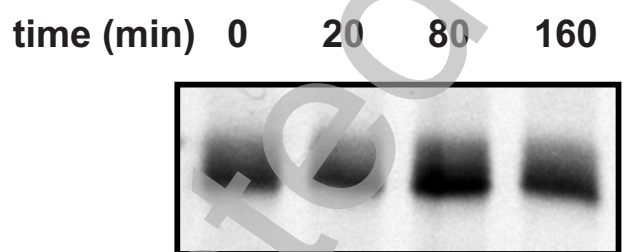

minus PSII

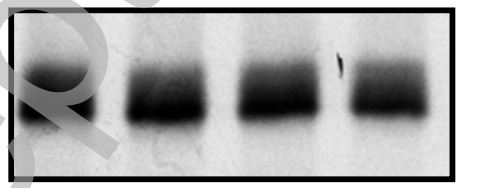

plus PSII

lane

$$
2 \quad 3 \quad 4
$$


Figure 7

A

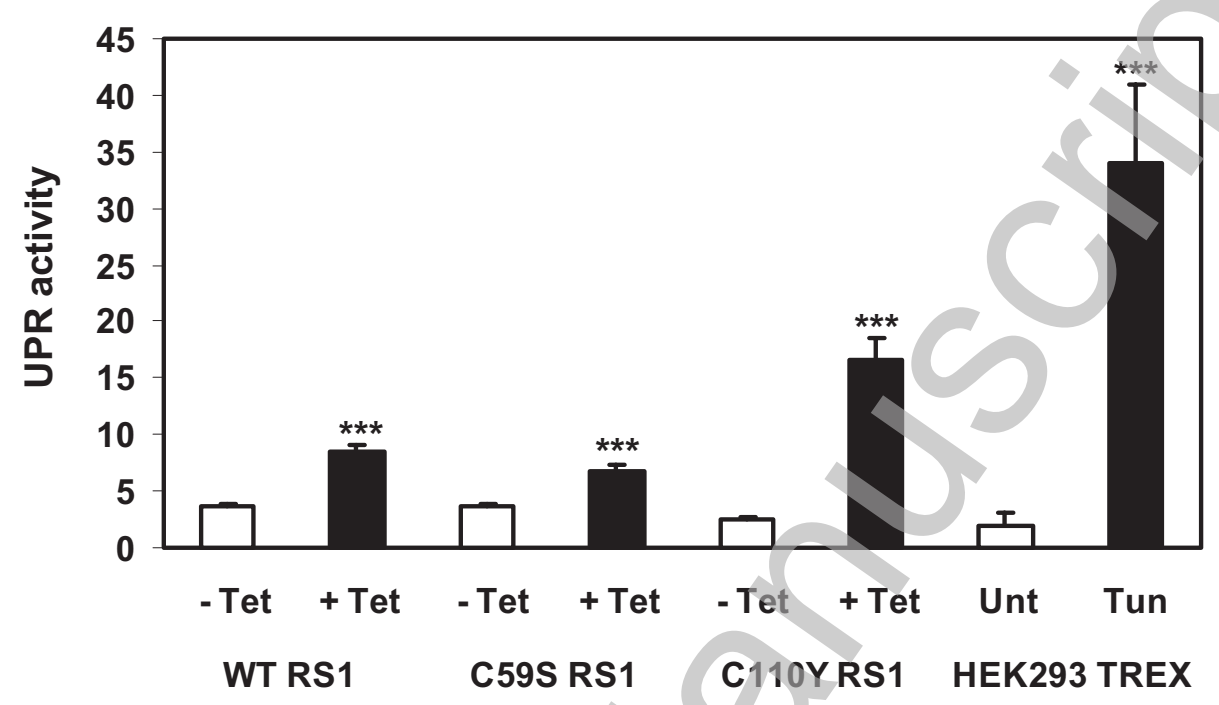

B

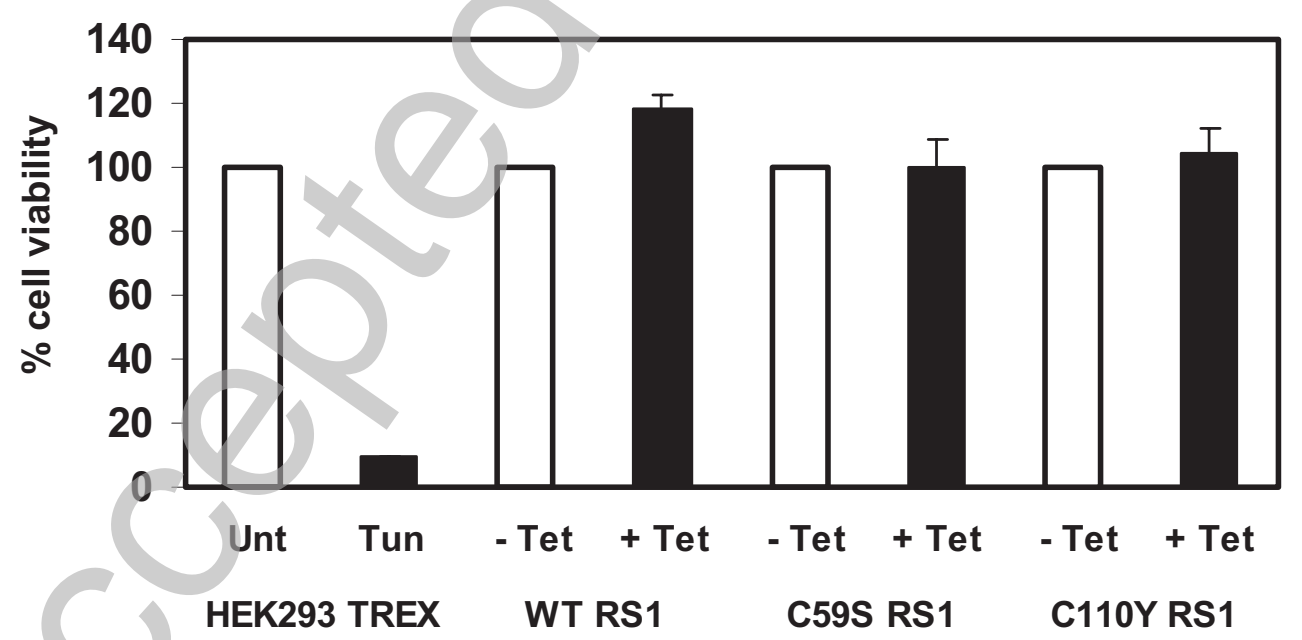

